\title{
Rapid clonal analysis of recurrent tuberculosis by direct MIRU-VNTR typing on stored isolates
}

\author{
Ana Martín, Marta Herránz, María Jesús Ruiz Serrano, Emilio Bouza and \\ Darío García de Viedma*
}

\begin{abstract}
Address: Servicio de Microbiología y Enfermedades Infecciosas. Hospital General Universitario Gregorio Marañón, CIBER Enfermedades Respiratorias (CIBERES), Universidad Complutense, Madrid, Spain

Email: Ana Martín - anamartsan@yahoo.es; Marta Herránz - m_herranz01@hotmail.com; María Jesús Ruiz Serrano - mruiz@hgugm.salud.madrid.org; Emilio Bouza - ebouza@microb.net; Darío García de Viedma* - dgviedma@microb.net

* Corresponding author
\end{abstract}

Published: 30 July 2007

BMC Microbiology 2007, 7:73 doi:10.1186/147/-2180-7-73
Received: 25 April 2007

Accepted: 30 July 2007

This article is available from: http://www.biomedcentral.com/147I-2180/7/73

(C) 2007 Martín et al; licensee BioMed Central Ltd.

This is an Open Access article distributed under the terms of the Creative Commons Attribution License (http://creativecommons.org/licenses/by/2.0), which permits unrestricted use, distribution, and reproduction in any medium, provided the original work is properly cited.

\begin{abstract}
Background: The application of molecular tools to the analysis of tuberculosis has revealed examples of clonal complexity, such as exogenous reinfection, coinfection, microevolution or compartmentalization. The detection of clonal heterogeneity by standard genotyping approaches is laborious and often requires expertise. This restricts the rapid availability of Mycobacterium tuberculosis (MTB) genotypes for clinical or therapeutic decision-making. A new PCR-based technique, MIRU-VNTR, has made it possible to genotype MTB in a time frame close to real-time fingerprinting. Our purpose was to evaluate the capacity of this technique to provide clinicians with a rapid discrimination between reactivation and exogenous reinfection and whether MIRU-VNTR makes it possible to obtain data directly from stored MTB isolates from recurrent episodes.
\end{abstract}

Results: We detected differences, between the MIRUtypes of recurrent isolates in 38.5\% (5//3) of the cases studied. These included cases of i) exogenous reinfection, often with more resistant strains, ii) likely examples of microevolution, leading to the appearance of new clonal variants and iii) a combination of microevolution, coinfection and competition.

Conclusion: MIRU-VNTR rapidly obtained clinically useful genotyping data in a challenging situation, directly from stored MTB isolates without subculturing them or purifying their DNA. Our results also mean that MIRU-VNTR could be applied for easy, rapid and affordable massive screening of collections of stored MTB isolates, which could establish the real dimension of clonal heterogeneity in MTB infection.

\section{Background}

Until recently, infection by Mycobacterium tuberculosis (MTB) has been assumed to be clonally simple, and a tuberculosis $(\mathrm{TB})$ case was traditionally thought to be infected by a single MTB strain. The introduction of molecular biology tools into the clinical microbiology laboratory has shown that this infection is sometimes clonally complex. In this sense, fingerprinting of clinical cultures has revealed i) exogenous reinfection as a cause of recurrences more often than originally thought [1-5], ii) simultaneous coinfection with different MTB strains [4-7] iii) microevolution phenomena $[8,9]$ and iv) compart- 
mentalization of the infection, with different strains infecting different tissues $[9,10]$, or even independent lung sites [11].

Clonal complexity in TB is now well documented, and clinicians are increasingly requesting characterization of the strains involved in these cases, mainly to discriminate between reinfection and reactivation. However, RFLPbased or spoligotyping-based fingerprinting approaches do not provide results quickly enough to include MTB genotypes in the pool of the first-line microbiological data available to take clinical, therapeutic or epidemiological decisions.

A new PCR-based fingerprinting technique, MIRU-VNTR [12], has been considered a suitable alternative for the simple and rapid detection of clonal complexity in TB $[7,13,14]$. We verified whether MIRU-VNTR could provide a rapid answer in conditions mimicking a real clinical situation: when MTB is cultured from a patient who had a previous $\mathrm{TB}$ episode and the clinician demands the discrimination between reactivation and exogenous reinfection. We evaluated the efficiency of MIRU-VNTR for the rapid analysis of clonal complexity in recurrent cases directly from a collection of frozen-stored MTB isolates, without subculturing them or purifying DNA.

\section{Results}

We were able to analyze the sequential MTB isolates of 58 patients with two or more recurrent TB episodes (2-5 episodes separated by intervals of between 6 months and 5 years). Thirty-two stored MTB isolates from 13 representative cases of recurrences (Table 1) were selected for MIRU-VNTR genotyping.

We could amplify at least ten of the 12 MIRU targets from twenty-nine stored clinical isolates $(90.6 \%)$. In an additional case we were only able to obtain a fingerprint from six loci. For the remaining two isolates, no MIRU loci were amplified (corresponding to two isolates from the years 1997 and 2003) and for one of these, MTB was not recovered after subculture.

\section{Clonal analysis of recurrences}

We compared the genotypes of the recurrent isolates obtained directly from the stored isolates. We found different MIRU types in the recurrent isolates in 5 of the 13 patients (38.5\%; cases A, I, J, K, L) and identical MIRU types were detected for the remaining eight cases (Table 1 , Figures 1 and 2). A highly discriminatory MIRU set of 15 loci was also applied with these eight cases and identical patterns for the sequential episodes were again obtained (data not shown). In three of the patients with clonal differences the isolates differed in two or more loci, including one case whose sequential isolates differed in six loci.
For patients I, J, K, i.e. those with differences in two or more than two MIRU loci, the RFLP and spoligotyping analysis confirmed the participation of different MTB strains (reinfection) in the first and recurrent episodes (Figure 1). In addition, in two of these cases (I and $\mathrm{K}$ ) the susceptibility pattern of the sequential isolates changed from susceptible to resistant (Figure 1).

In patients $\mathrm{L}$ and $\mathrm{A}$, the differences between recurrent isolates were more subtle, and affected only one locus (Figure 2). RFLP and spoligotype showed identical patterns for these clonal variants. Therefore we considered that these cases could be microevolution phenomena and not true reinfections. Whereas case $\mathrm{L}$ was an example of likely simple microevolution, a much more complex situation was detected for case A (Figure 2). This patient was initially infected by a single strain. However, a clonal variant appeared in the second episode, and continued to coinfect the patient together with the parental clone. This coinfection by both clonal variants was detected in the second, third and fourth episodes and finally, the first variant was the only one recovered from the fifth episode. In this case we observed differences in susceptibility patterns for pyrazinamide in the sequential episodes (Figure 2).

\section{Discussion}

The introduction of molecular biology tools in the clinical setting has revealed that MTB infection is clonally complex and that exogenous reinfection [1-5], simultaneous coinfection [4-7], compartmentalization $[9,10]$ and microevolution $[8,9]$ are examples of this clonal complexity.

In the past, clinicians assumed that clonal complexity had no impact on the management of TB and it was considered to be merely a refinement of the microbiological analysis. However, there are reports of $\mathrm{TB}$ cases with clonal complexity in which the strains can differ in their susceptibility patterns $[11,15-17]$, infectivity [18], or ability to infect extrarespiratory sites $[9,10]$. This has alerted clinicians to the usefulness of accessing to advanced molecular microbiological analysis.

The procedures usually applied to detect clonal heterogeneity are based on low- intensity RFLP bands or on the analysis of multiple single MTB colonies from culture. They are cumbersome, require expertise and cannot provide a quick answer in a real clinical setting. Recently, a novel genotyping method, MIRU-VNTR [12], has been proposed as an alternative to simplify and optimize the detection of clonal complexity directly from culture $[13,19]$.

In this study, we evaluate the efficiency of MIRU-VNTR at providing a rapid answer to a frequent request by clini- 
Table I: Characteristics of the patients. IVDU: intravenous drug user

\begin{tabular}{|c|c|c|c|c|c|c|}
\hline PATIENT & SEX & AGE & RISK FACTORS & EPISODES* & $\begin{array}{c}\text { EVIDENCE OF } \\
\text { NON- } \\
\text { ADHERENCE TO } \\
\text { THERAPY }\end{array}$ & $\begin{array}{c}\text { CLONAL } \\
\text { HETEROGENEIT } \\
Y\end{array}$ \\
\hline A & Female & 44 & & $9(4)$ & + & + \\
\hline B & Male & 50 & IVDU & 2 & + & \\
\hline $\mathrm{C}$ & Male & 46 & & $4(2)$ & & \\
\hline $\mathrm{D}$ & Male & 45 & $\begin{array}{l}\text { HIV+, IVDU, } \\
\text { Prison }\end{array}$ & 2 & + & \\
\hline$E$ & Male & 28 & & 3 & + & \\
\hline $\mathrm{F}$ & Male & 57 & Alcoholism & $4(3)$ & & \\
\hline G & Male & 52 & Alcoholism & 2 & + & \\
\hline $\mathrm{H}$ & Male & 32 & HIV+, IVDU & 4 & + & \\
\hline 1 & Female & 31 & HIV+, IVDU & 2 & & + \\
\hline j & Male & 46 & HIV+, IVDU & 2 & & + \\
\hline $\mathrm{K}$ & Male & 46 & HIV+, Alcoholism & 2 & & + \\
\hline L & Male & 53 & $\begin{array}{l}\text { IVDU, Alcoholism, } \\
\text { Prison }\end{array}$ & $3(2)$ & & + \\
\hline$M$ & Male & 53 & & 2 & & \\
\hline
\end{tabular}

*: The number of episodes with MTB isolates that could be analyzed by MIRU-VNTR are indicated in brackets.

cians, the discrimination between reactivation and exogenous reinfection in recurrent cases. Recurrence is not an anecdotal aspect of $\mathrm{TB}$, and between 1 and $11 \%$ of cases have a second recurrent episode ( $7 \%$ in our context). Rapid identification of recurrences caused by exogenous reinfection could influence therapeutic and epidemiological decisions because susceptibility could be different and the patient should be considered a new case. Besides, when a case is assumed to be a relapse, rapid information on exogenous reinfection by a strain spreading in the community could indicate new recent-transmission routes and guarantee rigor in the assignation of clusters indicating ongoing transmission events.

We evaluated the efficiency of MIRU-VNTR in studying the clonal composition of a selection of recurrent cases by analyzing our collection of frozen-stored isolates directly, without subculturing them or purifying DNA for the molecular study. It is common in the clinical context that MTB is cultured from a patient who had a previous TB episode and the isolate from the first episode is available in the strain-collection of the microbiology department. However, it is required to subculture the stored isolate before performing the comparative analysis, which means a great delay. The design we evaluate offers the possibility of analyzing stored isolates directly, in order to rapidly discriminate between reactivation and exogenous reinfection.

For 29 of the 32 selected isolates we obtained data (in at least 10 of the 12 loci studied), which shows the high power of resolution of MIRU-VNTR typing in this chal- lenging setting. In some cases, certain loci failed to be amplified directly from the stored isolates, and they could be amplified from new subcultures; however we have preferred to show only the results obtained from the direct amplification of stored isolates. In the case of the two loci that could not be often amplified (loci 10 and 16), we had previously observed (data not shown) that they were less efficiently amplified even with purified DNA samples. A remarkable feature is that the analysis could be performed even with isolates stored over a long period (study period: 1990-2006) and most of them were successfully amplified.

The 13 patients in our study population yielded 5 cases with some degree of clonal heterogeneity. In three of these cases (patients I, J, K) changes in two or more loci were observed between the recurrent isolates, suggesting an exogenous reinfection with a different strain. These results were confirmed by the clearly different RFLPtypes and spoligotypes after subculturing and analyzing the same isolates. It is worth noting that in two of these three cases, reinfection was associated with a change in the susceptibility patterns, from a susceptible strain to an INH-resistant strain, which is highly relevant for therapy. The patients analyzed in this study were different to those selected in a previous report [5] (33\% reinfections) and, again, the rates of reinfection $(23 \%)$ were higher than expected for a context with a moderate incidence of TB. The good correlation between VNTR-MIRU data and RFLP/spoligotyping indicated that differences in a small number of loci (in our case, two) could identify different strains without requiring standard genotyping analysis. In 


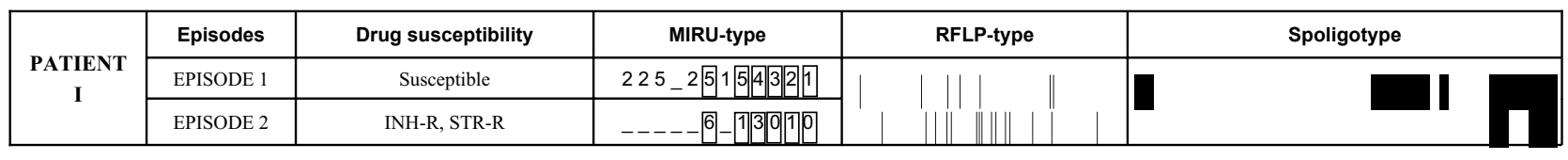

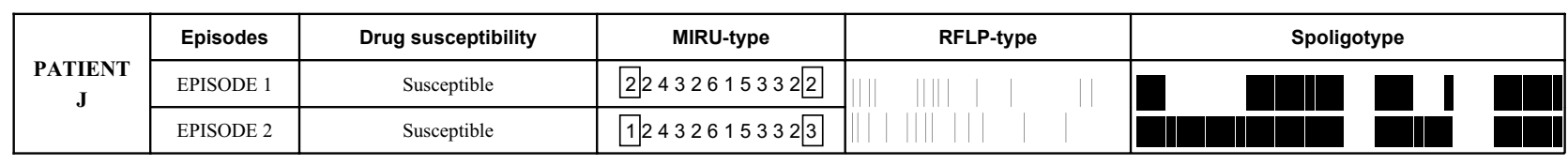

\begin{tabular}{|c|c|c|c|c|c|}
\hline \multirow{3}{*}{$\begin{array}{c}\text { PATIENT } \\
\text { K }\end{array}$} & Episodes & Drug susceptibility & MIRU-type & RFLP-type & Spoligotype \\
\hline & EPISODE 1 & Susceptible & $225-13153322$ & & \\
\hline & EPISODE 2 & INH-R & 223 322 153322 & & \\
\hline
\end{tabular}

Figure I

Susceptibility and genotyping features of the isolates from cases with reinfections. Differences in the MIRUtypes are boxed

this sense, a recent study in Shanghai [20] found that differences even in only one tandem repeat in a single MIRU locus enabled the identification of reinfections that were later confirmed by RFLP.

Although this study analyzed recurrent cases to distinguish reinfection from reactivation we found indirectly that the MIRU-VNTR approach succeeded in detecting clonal complexity other than reinfection. We identified another two cases with clonal heterogeneity (patients A and $\mathrm{L}$ ) but only subtle differences in one locus were detected. One of the cases (L) had only 6 bands in the RFLPtype and it could be a non clonal case [21]. However, these patients could also be likely examples of microevolutions leading to the appearance of clonal variants more than examples of reinfections, although appearance of clonal variants linked to MIRU-VNTR microevolution events has been found to be rather infrequent in other studies (2-6\% of the clonal cases)[22]. These recurrent isolates shared identical RFLP and spoligotypes which suggests that MIRU-VNTR has a higher sensitivity for detecting subtle genetic differences, although other studies [14] have shown that MIRU failed to detect subtle differences in RFLPtypes. The appearance of clonal variants in the context of an infection is not usually considered relevant but we report that it could be significant as seen in a case infected by two clonal variants with high genotypic similarity but with different infective behaviours; only one of them was able to infect the CNS whereas the other was restricted to the respiratory site [9]. The potential differential ability to cross the blood-brain barrier between both coinfecting variants is now being analyzed.

The situation of one of the patients whom clonal variants were found (case A) was complex, with a likely combination of microevolution, coinfection and competition. Here, our results could indicate that from an initial strain responsible for the first episode, another clonal variant was originated by genetic drift. The appearance of this second clone could be associated with the acquisition of resistance to pyrazinamide. Both clonal variants coinfected the patient over four years and finally, the original clone competed with the microevolved clone and the infection recovered its initial clonal homogeneity, with only the initial clone infecting the patient. However, it could also be considered that the absence of the second clone in the last specimen was caused by a sampling effect. Anyway, this interesting case shows that MTB infection could be a very dynamic process in clonal terms.

Our sample might be considered too small to enable clonal heterogeneity to be detected, nevertheless we detected a high percentage of these cases which suggests that clonal variability could be detected more frequently 


\begin{tabular}{|c|c|c|c|c|c|}
\hline \multirow{3}{*}{$\begin{array}{c}\text { PATIENT } \\
\text { L }\end{array}$} & Episodes & Drug susceptibility & MIRU-type & \multirow{3}{*}{ 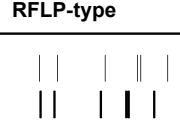 } & \multirow[t]{2}{*}{ Spoligotype } \\
\hline & EPISODE 1 & Susceptible & $223 \_26153323$ & & \\
\hline & EPISODE 2 & Susceptible & 223 _ 26153322 & & \\
\hline
\end{tabular}

\begin{tabular}{|c|c|c|c|c|c|}
\hline \multirow{6}{*}{$\begin{array}{c}\text { PATIENT } \\
\text { A }\end{array}$} & Episodes & Drug susceptibility & MIRU-type & \multirow{2}{*}{$\begin{array}{c}\text { RFLP-type } \\
\text { Not available }\end{array}$} & \multirow{2}{*}{$\begin{array}{r}\text { Spoligotype } \\
\text { Not available }\end{array}$} \\
\hline & EPISODE 1 & INH-R, RIF-R & 223525153322 & & \\
\hline & EPISODE 2 & INH-R, RIF-R & $223525 / 6153322$ & & \\
\hline & EPISODE 3 & INH-R, RIF-R, PZA-R & $223525 / 6153322$ & & \\
\hline & EPISODE 4 & INH-R, RIF-R, PZA-R & $223525 / 6153322$ & & \\
\hline & EPISODE 5 & INH-R, RIF-R, PZA-R & 223525153322 & & \\
\hline
\end{tabular}

\section{Figure 2}

Susceptibility and genotyping features from cases with subtle changes in the clonal composition of the isolates. Differences in the MIRUtypes are boxed

than expected. Moreover, the clonal complexity detected in this study reveals only a part of the real situation due to a potential underdetection of clonal heterogeneity caused by i) the fact that we could not obtain information from two loci in many cases, ii) the notion that MIRU 12 has low discriminatory power compared with a recently developed version with 15 loci $[23,24]$, and iii) the potential selection of certain MTB clones that could be present in clinical specimens but that could be counterselected and masked after culture.

\section{Conclusion}

In summary, MIRU-VNTR is an efficient technique for studying the clonal composition of MTB even in challenging laboratory circumstances such as the direct analysis of frozen-stored isolates without subculturing them or purifying DNA. It offers the possibility of rapidly answering clinical requests for identification of exogenous reinfection, reactivation or coinfection during the infection of patients with recurrent TB.

\section{Methods}

\section{Patients}

We analyzed patients from the general population with two or more TB episodes (MTB-positive culture) at least six months apart, during the period 1990-2006. From the 3237 cases with frozen-stored MTB clinical isolates for that period of time, 58 (1.8\%) were recurrent cases. From these, we selected 13 cases (all autochthonous Spanish cases) that were representative of a different number of episodes with different intervals between episodes. All but one (urine, case J) corresponded to respiratory samples.

\section{Samples}

The stored frozen samples contained two loopfuls of MTB culture suspended in $1.5 \mathrm{ml}$ of storage medium (7H9 Middlebrook $4.7 \mathrm{~g} / \mathrm{ml}$, sucrose 5\%, glycerin 2\%, OADC $10 \%)$. The frozen samples had been stored at $-70 \mathrm{C}$ from culture until analysis.

\section{MIRU-VNTR typing}

DNA was extracted by sonication in a water-bath sonicator (GenProbe) as described elsewhere [25]. Between 500 and $700 \mu \mathrm{l}$ of the stored culture was sonicated for seven minutes in the presence of 106-micrometers glass beads (SIGMA). DNA was not purified. 5 microliters of the crude extract was used for the typing assays by MIRU-VNTR using the 12-loci format [12]. Amplified products were run in a 2\% MS8 agarose gel (Pronadisa, Madrid, Spain) for 16.5 hours at $75 \mathrm{~V}$ to calculate the number of tandem repeats for each locus.

The potential involvement of laboratory cross-contamination in the misassigment of genotypes was ruled out by comparing the genotypes of all the isolates coprocessed (within a 3-day period) in the laboratory for each of the samples.

\section{IS6 I I O-RFLP and spoligotyping}

For the cases in which MIRUtyping detected genotypic differences between the isolates from the recurrent episodes, 
additional typing by IS6110-RFLP and spoligotyping was performed. For this purpose, the stored clinical MTB isolates were subcultured in Lowenstein-Jensen slants for three weeks, DNA was purified from the culture and RFLP and spoligotyping were performed following standard procedures $[26,27]$. Computer-assisted analysis of fingerprints was carried out using Bionumeric 5.1 software.

\section{Susceptibility test}

Susceptibility testing against isoniazid, rifampin, streptomycin, pyrazinamide and ethambutol was performed using the mycobacterial growth indicator SIRE system (Becton Dickinson).

\section{Authors' contributions}

Ana Martín: She has performed all the experimental assays and the MIRUtyping, she has analyzed the results and produced the first version of the MS

Marta Herránz: She has done the RFLP and spoligotyping

María Jesús Ruiz Serrano: She has done all the microbiological procedures

Emilio Bouza: He has revised critically the final version of the MS

Darío García de Viedma*: He has designed the study, supervised all experimental work, analyzed the results, corrected and produced the final version of the MS

\section{Acknowledgements}

We thank Sofia Samper (Hospital Universitario Miguel Servet, Zaragoza, Spain) for the MIRU data from the first episode of case A. We are indebted to Thomas O'Boyle for proofreading and editing the manuscript. A.M is receptor of a grant from Comunidad de Madrid cofinanced by the European Social Fund (Order n 5297/2006)

This study was partially financed by Fondo de Investigaciones Sanitarias (FIS030654; FIS060882) and by Instituto de Salud Carlos III (CIBER

Enfermedades Respiratorias CB06/06/0058). Potential conflict of interest: No conflict for all authors.

\section{References}

I. van Rie A, Warren R, Richardson M, Victor TC, Gie RP, Enarson DA, Beyers N, van Helden PD: Exogenous reinfection as a cause of recurrent tuberculosis after curative treatment. $\mathrm{N}$ EnglJ Med 1999, 34 I( (16): I I74-1 I79.

2. Bandera A, Gori A, Catozzi L, Degli Esposti A, Marchetti G, Molteni C, Ferrario G, Codecasa L, Penati V, Matteelli A, Franzetti F: Molecular epidemiology study of exogenous reinfection in an area with a low incidence of tuberculosis. J Clin Microbiol 200I, 39(6):2213-2218.

3. Caminero JA, Pena MJ, Campos-Herrero MI, Rodriguez JC, Afonso O, Martin C, Pavon JM, Torres MJ, Burgos M, Cabrera P, Small PM, Enarson DA: Exogenous reinfection with tuberculosis on a European island with a moderate incidence of disease. Am J Respir Crit Care Med 200I, 163(3 Pt I):717-720.

4. Chaves F, Dronda F, Alonso-Sanz M, Noriega AR: Evidence of exogenous reinfection and mixed infection with more than one strain of Mycobacterium tuberculosis among Spanish HIV-infected inmates. Aids 1999, I3(5):6I5-620.

5. Garcia de Viedma D, Marin M, Hernangomez S, Diaz M, Ruiz Serrano $M$ J, Alcala L, Bouza $E$ : Tuberculosis recurrences: reinfection plays a role in a population whose clinical/epidemiological characteristics do not favor reinfection. Arch Intern Med 2002, 162(16): 1873-1879.

6. Warren RM, Victor TC, Streicher EM, Richardson M, Beyers N, van Pittius NC, van Helden PD: Patients with active tuberculosis often have different strains in the same sputum specimen. Am J Respir Crit Care Med 2004, 169(5):610-614.

7. Shamputa IC, Jugheli L, Sadradze N, Willery E, Portaels F, Supply P, Rigouts L: Mixed infection and clonal representativeness of a single sputum sample in tuberculosis patients from a penitentiary hospital in Georgia. Respir Res 2006, 7:99.

8. Glynn JR, Yates MD, Crampin AC, Ngwira BM, Mwaungulu FD, Black GF, Chaguluka SD, Mwafulirwa DT, Floyd S, Murphy C, Drobniewski FA, Fine PE: DNA fingerprint changes in tuberculosis: reinfection, evolution, or laboratory error? J Infect Dis 2004, 190(6): I I 58-1I66.

9. Garcia de Viedma D, Marin M, Andres S, Lorenzo G, Ruiz-Serrano MJ, Bouza E: Complex clonal features in an mycobacterium tuberculosis infection in a two-year-old child. Pediatr Infect Dis J 2006, 25(5):457-459.

10. Garcia de Viedma D, Marin M, Ruiz Serrano MJ, Alcala L, Bouza E: Polyclonal and compartmentalized infection by Mycobacterium tuberculosis in patients with both respiratory and extrarespiratory involvement. J Infect Dis 2003, I 87(4):695-699.

II. Kaplan G, Post FA, Moreira AL, Wainwright H, Kreiswirth BN, Tanverdi M, Mathema B, Ramaswamy SV, Walther G, Steyn LM, Barry CE 3rd, Bekker LG: Mycobacterium tuberculosis growth at the cavity surface: a microenvironment with failed immunity. Infect Immun 2003, 7 I ( I 2):7099-7I 08.

12. Supply P, Lesjean S, Savine E, Kremer K, van Soolingen D, Locht C: Automated high-throughput genotyping for study of global epidemiology of Mycobacterium tuberculosis based on mycobacterial interspersed repetitive units. J Clin Microbiol 200I, 39( I 0):3563-357I.

13. Garcia de Viedma D, Alonso Rodriguez N, Andres S, Ruiz Serrano MJ, Bouza E: Characterization of clonal complexity in tuberculosis by mycobacterial interspersed repetitive unit-variablenumber tandem repeat typing. I Clin Microbiol 2005, 43(II):5660-5664.

14. Shamputa IC, Rigouts L, Eyongeta LA, El Aila NA, van Deun A, Salim $\mathrm{AH}$, Willery E, Locht C, Supply P, Portaels F: Genotypic and phenotypic heterogeneity among Mycobacterium tuberculosis isolates from pulmonary tuberculosis patients. J Clin Microbiol 2004, 42(I 2):5528-5536.

15. Post FA, Willcox PA, Mathema B, Steyn LM, Shean K, Ramaswamy SV, Graviss EA, Shashkina E, Kreiswirth BN, Kaplan G: Genetic polymorphism in Mycobacterium tuberculosis isolates from patients with chronic multidrug-resistant tuberculosis. J Infect Dis 2004, I 90 (I):99-106.

16. Turett GS, Fazal BA, Justman JE, Alland D, Duncalf RM, Telzak EE: Exogenous reinfection with multidrug-resistant Mycobacterium tuberculosis. Clin Infect Dis 1997, 24(3):5।3-5।4.

17. Horn DL, Hewlett D Jr., Haas WH, Butler WR, Alfalla C, Tan E, Levine A, Nayak A, Opal SM: Superinfection with rifampin-isoniazid-streptomycin-ethambutol (RISE)-resistant tuberculosis in three patients with AIDS: confirmation by polymerase chain reaction fingerprinting. Ann Intern Med 1994, I 2 I(2): I I5-II6.

18. Garcia de Viedma D, Lorenzo G, Cardona PJ, Rodriguez NA, Gordillo $S$, Serrano MJ, Bouza E: Association between the infectivity of Mycobacterium tuberculosis strains and their efficiency for extrarespiratory infection. J Infect Dis 2005, 1 92( ( 2):2059-2065.

19. Allix C, Supply P, Fauville-Dufaux M: Utility of fast mycobacterial interspersed repetitive unit-variable number tandem repeat genotyping in clinical mycobacteriological analysis. Clin Infect Dis 2004, 39(6):783-789.

20. Shen G, Xue Z, Shen X, Sun B, Gui X, Shen M, Mei J, Gao Q: Recurrent tuberculosis and exogenous reinfection, Shangai, China. Emerg Infect Dis 2006, I 2(II): I776-1778.

21. Cowan LS, Diem L, Monson T, Wand P, Temporado D, Oemig TV, Crawford JT: Evaluation of a two-step approach for largescale, prospective genotyping of Mycobacterium tuberculo- 
sis isolates in the United States. J Clin Microbiol 2005, 43(2):688-695.

22. Savine E, Warren RM, van der Spuy GD, Beyers N, van Helden PD, Locht C, Supply P: Stability of variable-number tandem repeats of mycobacterial interspersed repetitive units from I 2 loci in serial isolates of Mycobacterium tuberculosis. J Clin Microbiol 2002, 40( I 2):456 I-4566.

23. Supply P, Allix C, Lesjean S, Cardoso-Oelemann M, Rusch-Gerdes S, Willery E, Savine E, de Haas P, van Deutekom H, Roring S, Bifani P, Kurepina N, Kreiswirth B, Sola C, Rastogi N, Vatin V, Gutierrez MC, Fauville M, Niemann S, Skuce R, Kremer K, Locht C, van Soolingen D: Proposal for standardization of optimized Mycobacterial Interspersed Repetitive Unit-Variable Number Tandem Repeat typing of Mycobacterium tuberculosis. J Clin Microbiol 2006, 44(I 2):4498-45 I0.

24. Allix C, Walravens K, Saegerman C, Godfroid J, Supply P, FauvilleDufaux M: Evaluation of the epidemiological relevance of variable-number tandem-repeat genotyping of Mycobacterium bovis and comparison of the method with IS6 II 10 restriction fragment length polymorphism analysis and spoligotyping. Clin Microbiol 2006, 44(6): 195I-1 962.

25. Garcia de Viedma D, Marin M, Ruiz MJ, Bouza E: Analysis of clonal composition of Mycobacterium tuberculosis isolates in primary infections in children. J Clin Microbiol 2004, 42(8):34|5-34|8.

26. van Embden JD, Cave MD, Crawford JT, Dale JW, Eisenach KD, Gicquel B, Hermans P, Martin C, McAdam R, Shinnick TM, et al:: Strain identification of Mycobacterium tuberculosis by DNA fingerprinting: recommendations for a standardized methodology. J Clin Microbiol I993, 3 I (2):406-409.

27. Kamerbeek J, Schouls L, Kolk A, van Agterveld M, van Soolingen D, Kuijper S, Bunschoten A, Molhuizen H, Shaw R, Goyal M, van Embden $\mathrm{J}$ : Simultaneous detection and strain differentiation of Mycobacterium tuberculosis for diagnosis and epidemiology. J Clin Microbiol 1997, 35(4):907-914.

Publish with Bio Med Central and every scientist can read your work free of charge

"BioMed Central will be the most significant development for disseminating the results of biomedical research in our lifetime. "

Sir Paul Nurse, Cancer Research UK

Your research papers will be:

- available free of charge to the entire biomedical community

- peer reviewed and published immediately upon acceptance

- cited in PubMed and archived on PubMed Central

- yours - you keep the copyright
BioMedcentral 